\title{
X-ray Signatures of Charge Transfer Reactions Involving Cold, Very Highly Charged lons
}

P. Beiersdorfer, R.E. Olson, L. Schweikhard, P. Liebisch, G.V. Brown, J. Crespo López-Urrutia, C.L. Harris, P.A. Neill, S.B. Utter, K. Widmann

This article was submitted to XXI International Conference on the Physics of Electron and Atomic Collision, Seudai, Japan, July 22-27, 1999

\section{September 10, 1999}




\section{DISCLAIMER}

This document was prepared as an account of work sponsored by an agency of the United States Government. Neither the United States Government nor the University of California nor any of their employees, makes any warranty, express or implied, or assumes any legal liability or responsibility for the accuracy, completeness, or usefulness of any information, apparatus, product, or process

disclosed, or represents that its use would not infringe privately owned rights. Reference herein to any specific commercial product, process, or service by trade name, trademark, manufacturer, or otherwise, does not necessarily constitute or imply its endorsement, recommendation, or favoring by the United States Government or the University of California. The views and opinions of authors expressed herein do not necessarily state or reflect those of the United States Government or the University of California, and shall not be used for advertising or product endorsement purposes. 


\title{
X-ray Signatures of Charge Transfer Reactions Involving Cold, Very Highly Charged Ions
}

\author{
P. Beiersdorfer ${ }^{a 1}$, R. E. Olson ${ }^{b}$, L. Schweikhard ${ }^{c}$, P. Liebisch ${ }^{c}$ \\ G. V. Brown ${ }^{a}$, J. Crespo López-Urrutia ${ }^{a}$, C. L. Harris ${ }^{d}$ \\ P. A. Neill ${ }^{a}$, S. B. Utter ${ }^{a}$, K. Widmann ${ }^{a}$ \\ ${ }^{a}$ Department of Physics, Lawrence Livermore National Laboratory, Livermore, CA 94550, USA \\ ${ }^{b}$ Department of Physics, University of Missouri-Rolla, Rolla, MO 65401, USA \\ ${ }^{c}$ Institut für Physik, Johannes Gutenberg-Universität, D-55099 Mainz, Germany \\ ${ }^{d}$ Department of Physics, University of Nevada Reno, Reno NV 89557, USA
}

\begin{abstract}
.
Charge transfer reactions involving highly charged ions up to $\mathrm{U}^{91+}$ are being studied using $\mathrm{x}$-ray spectroscopic techniques. 'The measurements are performed using ions produced in situ in a cylindrical trap with very low kinetic energy $(\leq 3 \mathrm{eV} / \mathrm{amu})$. 'The observed K-shell and L-shell x-ray emission show prominent features from high- $n$ levels decaying to the ground level. Such emission can serve as a diagnostic marker for charge transfer processes in plasmas, for example in cometary comae or in ion traps. The emission is enabled by electron capture into states with low orbital angular momentum, mainly $\ell=1$. The observed spectra, thus, provide a handle on determining the fraction of electron capture into low- $\ell$ states that can be used for testing theoretical models in the limit of low collision energy. The present paper summarizes the progress made to date.
\end{abstract}

\section{INTRODUCTION}

As an ion approaches a neutral atom or molecule, one or more electrons may transfer from the neutral to the ion, thereby lowering the ion's charge. This process, dubbed charge exchange or charge transfer, is an important process in situations when ions come in contact with neutrals. In laboratory plasmas this invariably happens near the plasma edge region, for example in the divertor region of magnetic fusion devices. It also happens when energetic neutral beams are used for plasma heating, or when the plasma is fueled by the injection of solid pellets. Highly charged ions circulating in a storage ring undergo charge exchange with background neutrals. Similarly, the ion storage time in a Penning trap is limited by charge

1) Email address: beiersdorfer@ịlnl.gov 
transfer reactions. An accurate description of charge transfer processes is thus not only important for understanding basic atomic processses but has important implications for describing and controlling laboratory plasmas and ion trapping. Low-energy collisions below about $100 \mathrm{eV} / \mathrm{amu}$, in particular, have now also become of great interest in describing non-terrestrial phenomena, such as the $\mathrm{x}$-ray emission from comets. Cometary $\mathrm{x}$-ray emission is presumed to emanate from the interaction between highly charged heavy ions in the solar wind and gases in the coma. The collision energy is thought to be on the order of about $50 \mathrm{eV} / \mathrm{amu}$.

Measurements of charge transfer have mostly focused on determining the total cross section of the interaction, as this is the fundamental quantity needed to describe ionization balance in plasmas or ion storage times in storage rings and traps. Such measurements involve detailed accounting of the number and type of particles involved in the process before and after a charge changing collision. Measurements of the charge exchange cross sections have been obtained this way for a variety of collision partners (e.g. [1-3]).

Another way to study charge transfer is by looking at the photons emitted in the reaction. Photon emission results from the fact that the electron is captured by the ion into an orbital with high principal quantum number $n$. As illustrated in Fig. 1, this electron will radiatively deexcite by sequentially giving off one or more photons:

$$
\begin{gathered}
A^{q+}+B^{0} \rightarrow A^{(q-1)+*}+B^{+} \\
A^{(q-1)+*} \rightarrow A^{(q-1)+* *}+h \nu_{1} \rightarrow A^{(q-1)+* *}+h \nu_{2} \text { etc. }
\end{gathered}
$$

Observations of photon emission have concentrated on visible light. This is because of the interest in magnetic fusion to spectroscopically observe charge transfer between energetic neutral beams and plasma ions [4-6]. In such high-energy collisions, the electron is captured into high angular momentum states, and deexcitation proceeds along the yrast chain where each deexcitation step reduces the angular momentum and principal quantum number by unity, as indicated in Fig. 1(a).

Charge transfer processes also produce $x$-ray emission. Even if the initial decay is from one high- $n$ state to a state of $n-1$ by giving off a photon in the visible, this process eventually will produce photons of higher and higher energy as $n$ gets smaller. Ions with an open $\mathrm{K}$-shell will give off a $\mathrm{K}$-shell $\mathrm{x}$ ray when the electron deexcites from the $n=2$ shell to $n=1$. X rays with even higher energy are produced if selection rules allow the electron to make the jump from higher $n$ shells to ground. This is the case, if the angular momentum of the captured electron plus that of other bound electrons of the ion, if any, differs by that of the ground level by 0 or \pm 1 (if the ground level has an angular momentum of 0 , then the excited state cannot differ by 0 ). Studies of the x-ray emission pattern produced in charge exchange thus provide information on the angular momentum values associated with the electron capture. 


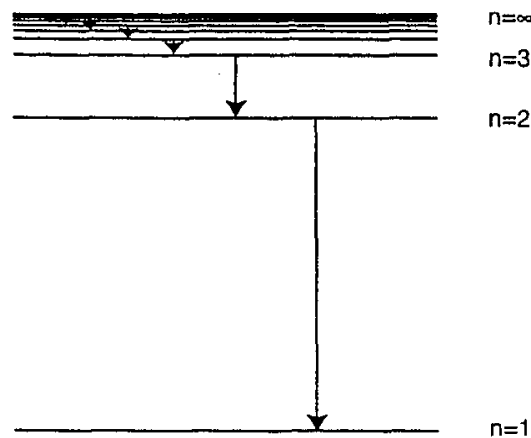

(a)

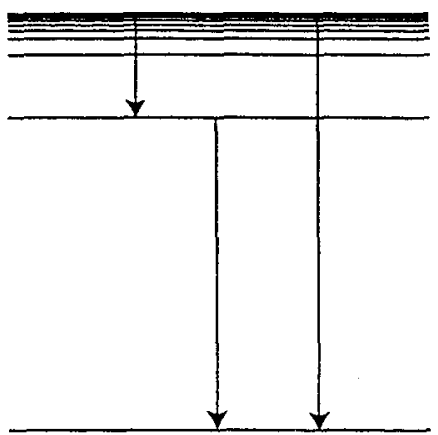

(b)

FIGURE 1. Radiative decay chain following electron capture into a high $n$ level. (a) transitions along the yrast chain $(\Delta \ell=1)$ after high-angular momentum capture in a high-energy collision; (b) transitions after $s, p$, and $d$ angular momentum capture in a low-energy collision.

Studies of the $\mathrm{x}$-ray emission from charge exchange have been made in the case of very energetic ion-atom collisions. For example, the x-ray emission of $50-360$ $\mathrm{MeV} / \mathrm{amu} \mathrm{U}^{92+}$ ions colliding with various atomic and molecular species in a gas-jet target was measured by Stölker et al. [7]. Very recently, work has begun to look at $\mathrm{x}$-rays emitted from ions colliding with neutrals at $0.3-7.5 \mathrm{keV}$ energies produced in ECR ion sources [8]. By contrast, $x$-ray measurements of very low-energy charge transfer collisions in the $\mathrm{eV}$ energy range have been made for several years now using the Livermore electron beam ion trap (EBIT) $[9,10]$.

The first $x$-ray measurements using the Tivermore FBIT were performed to demonstrate the utility of the device as an ion trap in the absence on an electron beam [9]. The electron beam was simply used to produce highly charged ions, but it is then turned off. In the absence of the electron beam, the ions are stored in a Penning trap defined by the $3-\mathrm{T}$ axial magnetic field and a 10 to $100 \mathrm{~V}$ potential on the end cap electrodes. The net effect is that the trap is filled in situ and thus does not suffer any transport losses. The trap can thus easily contain $10^{5}$ or $10^{6}$ ions of interest. This is in stark contrast to various schemes in which traps are filled from external ion sources where only $10^{\circ}$ to $10^{2}$ ions of interest are trapped at any given time [11]. This mode of operation of the Livermore EBIT was dubbed the magnetic trapping mode [12]. Depending on the base pressure and trapping potential, ion storage times as high as several seconds were found in these measurements $[9,12,13]$. The energy of the ions stored in the trap is limited by the depth of the potential well to values between about $0.5-50 \mathrm{eV} / \mathrm{amu}[14-16]$.

The very low collision energies that can be studied using the magnetic trapping mode enable the observation of phenomena that cannot be observed in high-energy collisions $250 \mathrm{eV} / \mathrm{amu}$ or higher. For example, the angular momentum values of the captured electron are no longer distributed statistically favoring high $\ell$ values but concentrate near the smaller values [17]. The result is that the $x$-ray emission changes character and on average moves toward higher energies. The change-over 


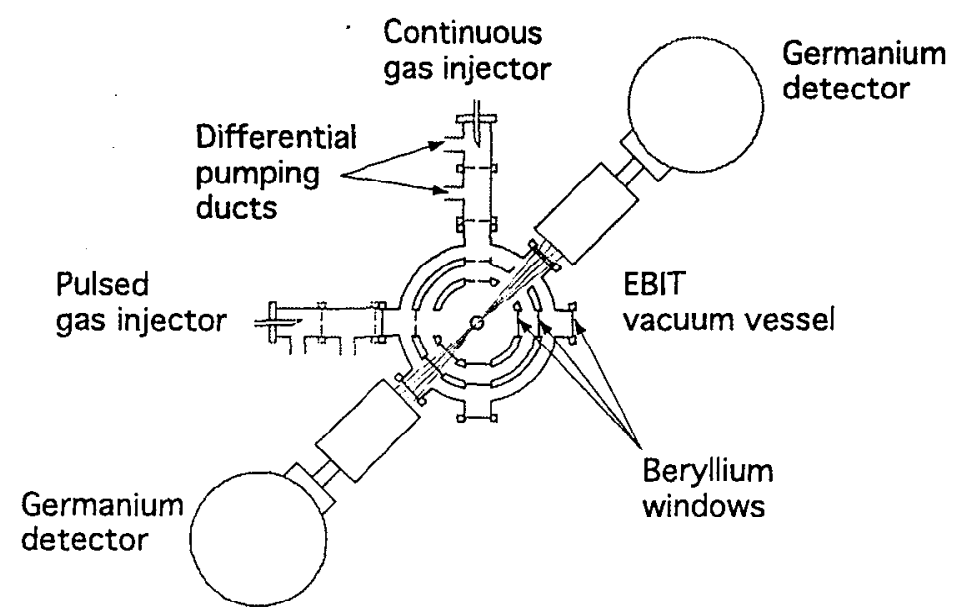

FIGURE 2. Cross-sectional view of the radial ports viewing the EBIT trap. The ports are used for gas injection and monitoring of the $\mathrm{x}$-ray emission from the trap.

between statistical and non-statistical population of angular momentum values is predicted to occur at collsion energies on the order of a few hundred eV/amu. This means that ion accelerator or even ECR-source based measurements cannot access this low-energy region, and sources like the Livermore EBIT need to be employed.

In the following we present an overview of the spectra obtained at low collision energy. The spectra are in part explained by classical trajectory Monte Carlo calculations of charge exchange reactions in ion-atom collisions. The possibility to study charge exchange reactions in a heretofore inaccessible regime continue to make this area of investigation novel and exciting.

\section{EXPERIMENTAL ARRANGEMENT}

The $\mathrm{x}$-ray emission produced in charge exchange reactions were recorded with a high-purity Ge detector, which looked directly into the trap, as illustrated in Fig. 2. A typical K-shell spectrum of krypton obtained during the magnetic mode is shown in Fig. 3. Only charge exchange into bare and hydrogenic ions contributes to the observed emission, as only these ions have the necessary K-shell vacancy to allow the emission of a $\mathrm{K}$-shell $\mathrm{x}$ ray. The spectrum not only contains features from the $2 \rightarrow 1$ transitions but also emission from the $n \rightarrow 1$ prompt decay indicating that a substantial fraction of electrons are captured into $\ell=1$ angular momentum states.

An L-shell emission spectrum of very highly charged uranium produced via charge exchange reactions is shown in Fig. 4. L-shell emission can only be produced if the capturing ion has one or more vacancies in the L-shell. A total of ten charge states (bare through fluorinelike) can thus contribute to the L-shell emission spectrum. In the present case, the charge balance was peaked near helium-, lithium-, and berylliumlike uranium. "No bare or hydrogenic uranium was produced. The ob- 


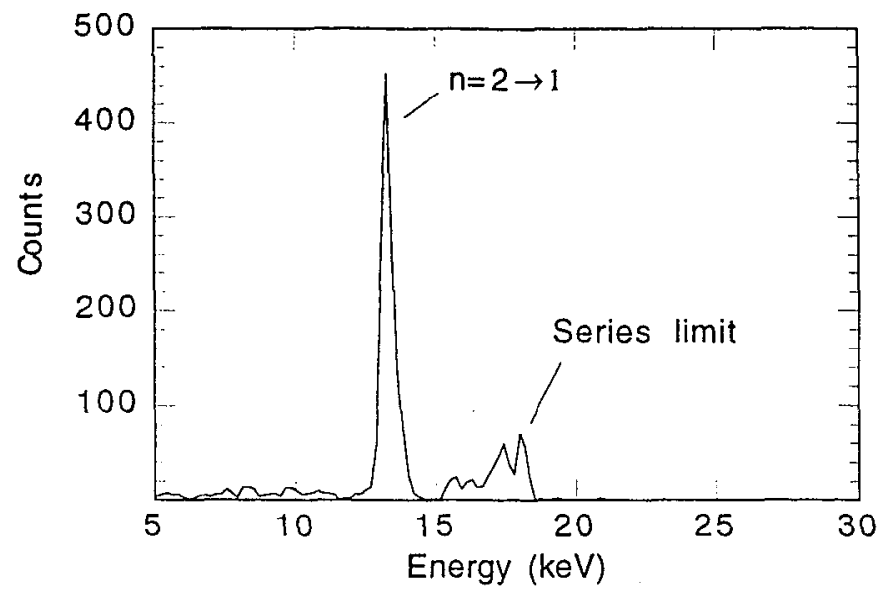

FIGURE 3. X-ray spectrum of K-shell krypton ions produced by charge exchange reactions of bare $\mathrm{Kr}^{36+}$ and hydrogenic $\mathrm{Kr}^{35+}$ ions with neutral krypton atoms.

served L-shell emission spectrum has one dominant peak. This peak is assigned to transitions of the type $3 s_{1 / 2} \rightarrow 2 p_{3 / 2}$. X-ray features at higher energy are seen as well, extending all the way to the series limit of $n \rightarrow 2 s$ prompt decay in lithiumlike uranium at $32.5 \mathrm{keV}$.

The charge-exchange-induced spectra can be compared to spectra produced by electron-impact excitation. In Fig. 4(b) we show the L-shell emission spectrum of uranium produced by direct electron impact recorded before the beam was turned off. Instead of one prominent line, three prominent lines are seen. These are assigned to transitions of the type $3 s_{1 / 2} \rightarrow 2 p_{3 / 2}, 3 d_{3 / 2} \rightarrow 2 p_{5 / 2}$, and $3 p_{1 / 2} \rightarrow 2 s_{1 / 2}$. The intensity of $x$-ray emission from the series limit $n \rightarrow 2$ is comparatively much weaker in Fig. 4(b) than Fig. 4(a).

Because the bacliground neutral pressure in the EBIT trap is low $\left(\leq 10^{-9}\right.$ torr) charge transfer processes involving background atoms happen on a rather long time scale. This was borne out by measuring the $\mathrm{x}$-ray signal produced as a function of time in the magnetic mode. As illustrated in Fig. 5, the x-ray signal decays on a time scale of hundreds of milliseconds in EBIT. Measurements on the high-energy SuperEBIT facility, which has an cven lower basc pressure ( $\leq 10^{-10}$ torr), have shown that charge transfer processes happen on time scales of multiple seconds $[9,12,13,18]$.

The rate of decay can be influenced by injecting gases at varying pressures. The $\mathrm{x}$-ray emission and ion storage time as a function of injection pressure was studied in Ref. [9]. Gas injection takes place by using a ballistic gas injector, as indicated in Fig. 2. We operate the injector either in a continuous mode [9] or in a pulsed mode [18].

In order to concentrate the charge exchange-induced $x$-ray emission into a a very short time interval, it is best to use the pulsed injection system. This is illustrated in Fig. 6, which shows the x-ray emission from the uranium L-shell as a function 

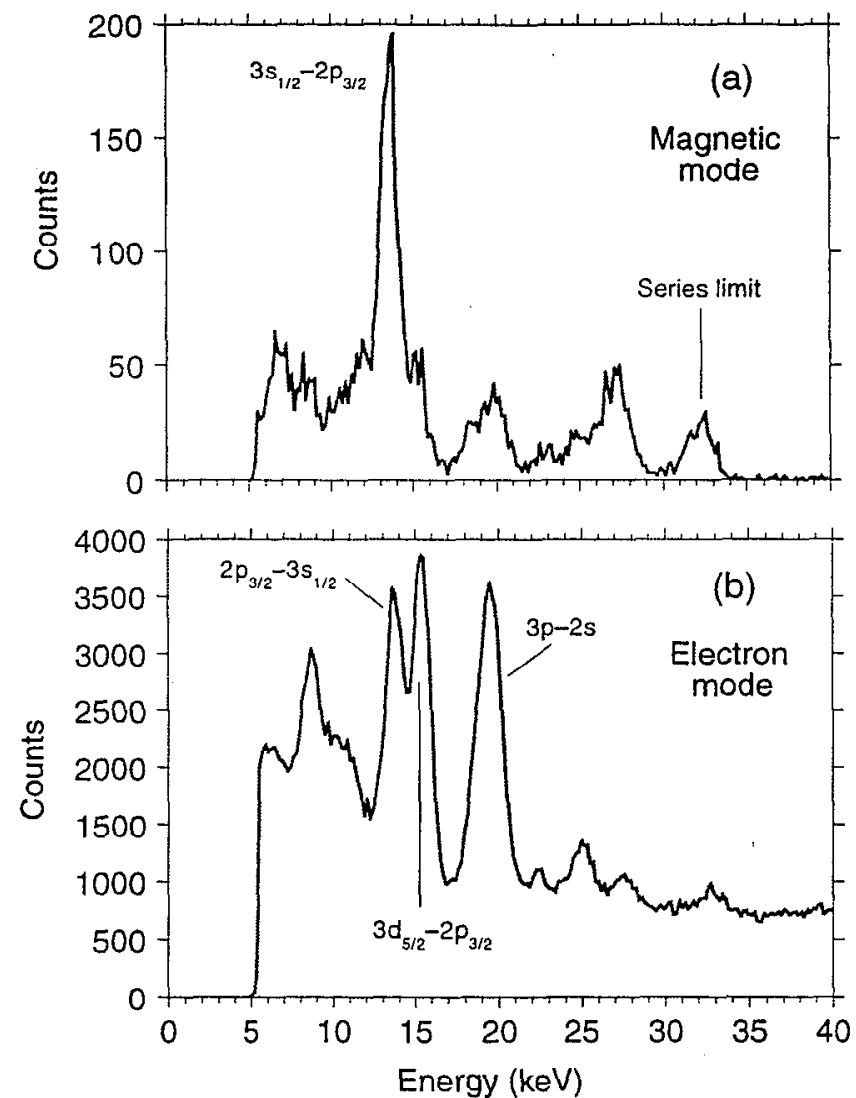

FIGURE 4. X-ray emission of L-shell uranium ions $\mathrm{U}^{q+}, q=83+, \ldots 90+$. (a) spectrum produced by charge exchange reactions with neon atoms; (b) spectrum produced by electron-impact excitation with a $120-\mathrm{keV}$ electron beam.

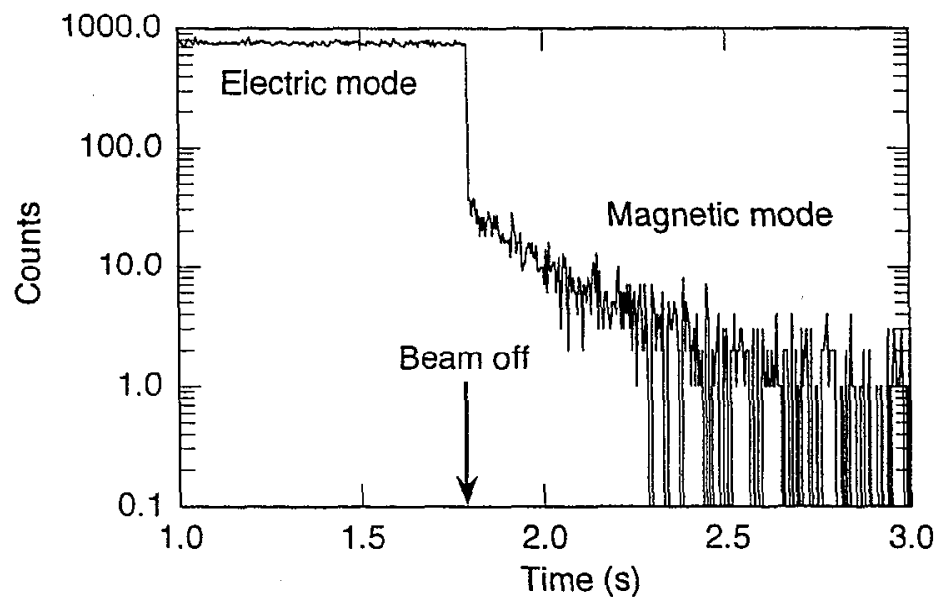

FIGURE 5. Time dependence of the K-shell $\mathrm{x}$-ray emission produced by charge transfer reaction of bare $\mathrm{Fe}^{26+}$ and hydrogenic $\mathrm{Fe}^{25+}$ ions with background gases. 


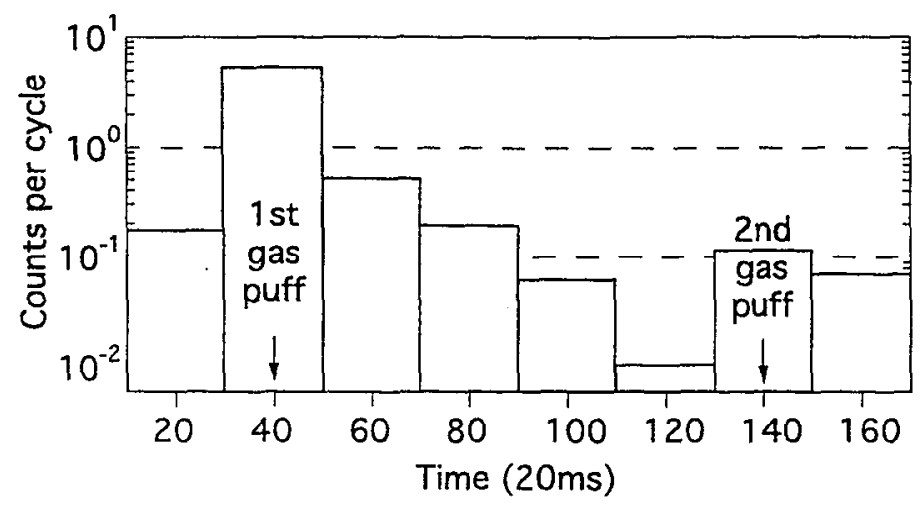

FIGURE 6. L-shell $x$-ray emission produced by charge transfer reactions of highly charged uranium ions $\mathrm{U}^{q+}, q=83+, \ldots 90+$ with neon introduced into the trap with a pulsed gas injector.

of time. A puff of neon gas is introduced into the trap via a fast acting $(\approx 20 \mu \mathrm{s})$ gas valve. The puff strongly increases the number of charge exchange reactions and concentrates the measured $x$-ray flux into a single time bin. A second puff, delayed by by $100 \mathrm{msec}$, is used to check whether all or most ions have recombined. It produces an x-ray flux that is fifty times less than that produced by the first puft. This demonstrates that the first puff indeed is sufficient to force charge exchange recombination of most uranium ions with an open L-shell in the trap.

\section{RESULTS AND DISCUSSION}

The observed spectral emission can be understood by noting that the impact parameter is very small for cold collisions. The spectra are, therefore, very different from those observed in high-energy collsions where the impact parameter is large. In high energy collisions, the values of angular momentum of the captured electron are statistically populated. This is not the case in low-energy collisions. A smaller impact parameter reduces the angular momentum value of the captured electron and limits it to a' small subset of values [17]. A dramatic illustration of this is given in Fig. 7, where we plot the predictions of classical trajectory Monte-Carlo calculations [19] for the capture cross section into a particular $\ell$ value in the reaction $\mathrm{Xe}^{54+}+\mathrm{H} \rightarrow\left(\mathrm{Xe}^{53+}\right)^{*}+\mathrm{H}^{+}$. The calculations were carried out for three different collision energies: $250 \mathrm{eV} / \mathrm{amu}, 2.5 \mathrm{eV} / \mathrm{amu}$, and $0.625 \mathrm{eV} / \mathrm{amu}$. The angular momentum values for $250 \mathrm{eV} / \mathrm{amu}$ are clearly statistical. This is not the case for the two lower energies. In the case of $2.5 \mathrm{eV} / \mathrm{amu}$ the distribution peaks at $\ell=5$. The peak drops to $\ell=3$ for the $0.625 \mathrm{eV} / \mathrm{amu}$ collision energy. The calculations clearly show that the $\mathrm{x}$-ray emission patterns will differ from the that produced by statistical population of the $\ell$ values only if the collision energy is below 250 $\mathrm{eV} / \mathrm{amu}$. Prompt $\mathrm{x}$-ray emission from transitions that proceed from the capturing level to ground is extremely weak in high-energy collisions. The prompt emission 


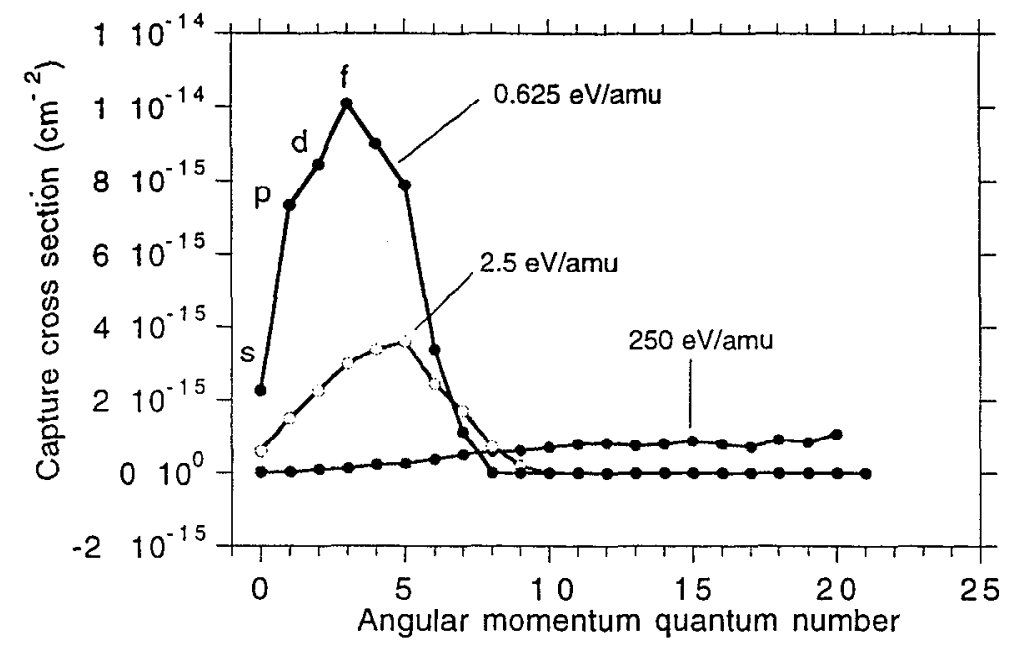

FIGURE 7. Predicted distribution of angular momentum values of electrons captured in the reaction $\mathrm{Xe}^{54+}+\mathrm{H} \rightarrow \mathrm{Xe}^{53+*}+\mathrm{H}^{+}$. Results of classical trajectory Monte Carlo calculations are shown for the different collision energies indicated.

that we observe can only be detected in the low collision energy regime inaccessible to measurements performed with ions from heavy-ion accelerators or ECR sources.

As the collision energy drops, the fraction of electrons captured into an $\ell=1$, i.e. $p$, state increases. $p$ electrons can decay directly to the $s$ ground level in hydrogenlike ions. Electrons captured with angular momentum $\ell=1$ produce prompt x-ray emission by decaying directly from the $n$ level into which they were captured to the $1 s$ grond level. Electrons with other angular momentum values will deexcited via intermediate steps, typically by ultimately producing a $n=2 \rightarrow$ $n=1 \mathrm{x}$ ray. The ratio of the intensity of the prompt $\mathrm{x}$ rays from high $n$ levels to the intensity of the $\mathrm{x}$ rays from the $n=2$ level, therefore, gives a measure of the fraction of electrons captured into $\ell=1$ angular momentum states. In Fig. 8 we plot the Monte Carlo prediction for this ratio. The figure clearly shows the increase in the amount of prompt $x$ rays as with decreasing collision energy.

Emission from high- $n$ levels is not only seen in K-shell spectra but also in the Lshell spectra. This was already illustrated in Fig. 4. The intensity of this emission is larger than it is in a comparable spectrum produced by electron-impact excitation. A quantitative measure of this enhancement is given in Table I in the case of B-like $\mathrm{Fe}^{21+}$. This ion has a partially open L-shell; the $2 s$ subshell is, however, filled. The comparison between the emission produced by electron-impact excitation and that produced by charge exchange shows that the emission from the $n=7$ level, which is most likely to capture a charge exchange electron, is enhanced by about a factor of five. Like in the case of $K$-shell ions, the enhancement can serve as a diagnostic indicator that charge-transfer processes contribute to or even dominate the $\mathrm{x}$-ray production. 


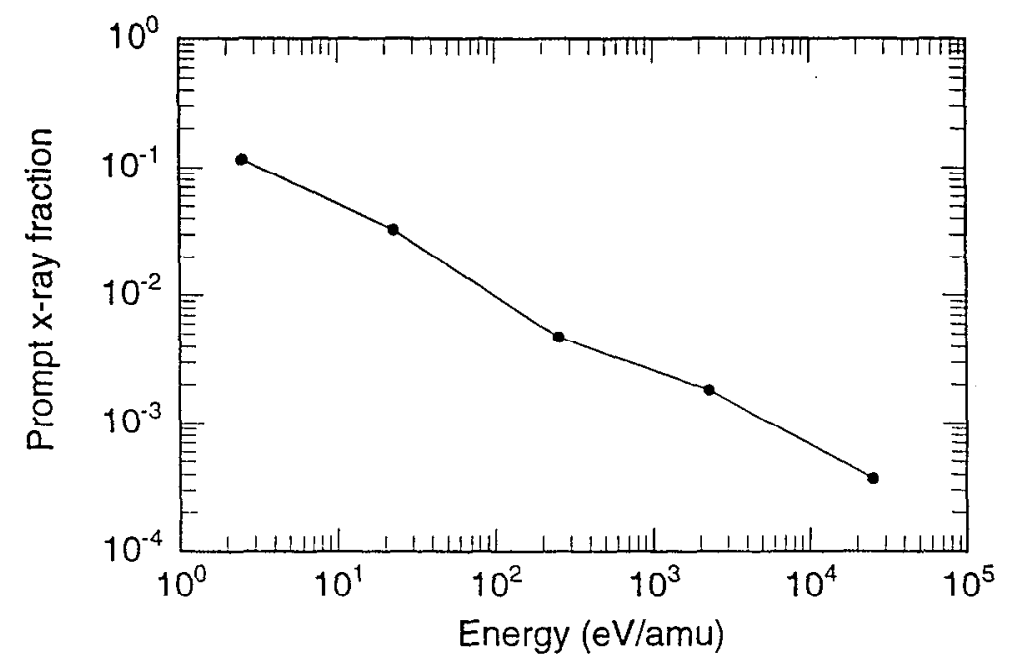

FIGURE 8. Fraction of emitted $\mathrm{x}$ rays from prompt radiative decay $n p \rightarrow 1 s$ as a function of collision energy. The results shown are for the process $\mathrm{Xe}^{54+}$ $+\mathrm{H} \rightarrow \mathrm{Xe}^{53+*}+\mathrm{H}^{+}$.

\section{CONCLUSION}

Using the unique capabilities afforded by operating the Livermore electron beam ion trap in the magnetic trapping mode, it was possible to study charge transfer reactions in a regime that was heretofore inaccessible to experimental scrutiny. Direct line-of-sight access to the trap allowed the recording of the x-ray emission produced in the reactions. X-ray emission patterns were observed that were different from the high-energy limit observed using ions from ECR sources or heavy-ion accelerators. We showed that "high" energy refers to energies $\geq 250 \mathrm{eV} / \mathrm{amu}$. The emitted x-ray pattern above this limit is virtually independent of collision energy. Below this energy limit, the angular momentum of the captured electron changes with energy resulting in a change of the $\mathrm{x}$-ray emission pattern. By studying this emission pattern, measurements allow us to determine the angular momentum values of the captured electron states. In particular, we can determine the fraction of capture into a $p$ state directly from the prompt $\mathrm{x}$-ray emission in $\mathrm{K}$-shell spectra. Studies are now under way to exploit this fact for detailed testing of atomic calculations and for developing spetral plasma diagnostics.

TABLE 1. Results from L-shell measurements of boronlike $\mathrm{Fe}^{21+}$. The results are normalized to the intensity of the $3 \rightarrow 2 \times$ rays.

\begin{tabular}{|c|c|c|}
\hline Transition & $\begin{array}{c}\text { Intensity } \\
\text { Electron-Impact Excitation }\end{array}$ & $\begin{array}{c}\text { Intensity } \\
\text { Charge-Transfer Excitation }\end{array}$ \\
\hline $3 \rightarrow 2$ & 100 & 100 \\
\hline $7 \rightarrow 2$ & 12.2 & 60 \\
\hline Series limit & 6.2 & 26 \\
\hline
\end{tabular}

